\title{
Efavirenz and Lopinavir Levels in HIV-Infected Women and Their Nursing Infants, in Mali
}

\author{
Aboubacar Alassane Oumar, Kadiatou Bagayoko-Maiga, Aliou Bahachimi, Mamoudou Maiga, \\ Marie-Christine Cere, Zoumana Diarra, Etienne Chatelut, Mariam Sylla, Robert Leo Murphy, \\ Sounkalo Dao, and Peggy Gandia
}

Pharmacokinetics and Toxicology Laboratory, Federative Institute of Biology, Purpan Hospital University, Toulouse, France (A.A.O., M.-C.C., P.G.); Institut National de la Sante et de la Recherche Medicale, Unité Mixte de Recherche 1037, Pharmacology and Pharmacogenetics Laboratory, University Institute of Cancer Oncopôle, Toulouse, France (A.A.O., E.C.); HIV/Tuberculosis Research and Training Center, University of Science, Techniques and Technologies, Bamako, Mali (A.A.O., A.B., M.M., S.D.); Center for Global Health, Northwestern University, Chicago, Illinois (M.M., R.L.M.); Department of Pediatric, University Hospital of Gabriel Touré, Bamako, Mali (K.B.-M., M.S.); Referral Health Center V, Bamako, Mali (Z.D.); and Institut National de la Recherche Agronomique Unité Mixte de Recherche 1331-TOXALIM, Veterinary School of Toulouse, Toulouse, France (P.G.)

Received April 12, 2018; accepted June 29, 2018

\begin{abstract}
Limited data are currently available on antiretroviral pharmacokinetics in breast milk (BM) and in breastfed infants' blood. To explore these parameters in patients in Mali, we measured plasma antiretroviral levels in human immunodeficiency virus (HIV)-infected mothers and their breastfed infants over 6 months. We specifically analyzed the concentrations of efavirenz (EFV) and lopinavir (LPV) in the plasma of mothers living with HIV and their breastfed infants. Blood samples were collected at delivery and at month 1, 3, and 6 postpartum. EFV and LPV concentrations were measured by liquid chromatography-tandem mass spectrometry. HIV-1 RNA load was measured by Abbott M2000RT RealTime System at delivery and 6 months postpartum for mothers, and at 3 and 6 months postbirth for infants. The median duration of antiretroviral therapy at study inclusion
\end{abstract}

was 57 months [interquartile range (IQR), 0-168 months]. The median EFV ratios of infant plasma/maternal plasma (MP) were 0.057 at month $1,0.072$ at month 3 , and 0.048 at month 6 . During the study period, the median BM/MP ratio of EFV was 1.16 (IQR, 0.96-20.62), which corresponds to a relative infant dose of $2.46 \%$ of the recommended weight-adjusted pediatric EFV dose at month 6 . The apparent infant clearance of EFV was $0.146 \mathrm{I} / \mathrm{h}$ per kilogram at month 6 . The LPV concentrations in the plasma of all infants were undetectable. No drug-related adverse reaction or toxicity was observed in any of the infants. The two women who presented a viral load of $>50$ copies $/ \mathrm{ml}$ at month 6 had undetectable plasma drug concentrations at the same period. This study showed that breastfed infants received a low level of EFV but not LPV from their treated mothers.
This work was supported by University of Bamako Fund, Mali, as part of funding for A.A.O.'s effort, (Grant 2013-04); The Richard and Susan Kiphart Northwestern Global Health Research Fund (Grant 2015-01), and the Toulouse University Hospital agency (ARS) (Grant 2015-01). No potential conflicts of interest relevant to this article are reported.

Part of this work has been presented as follows: Oumar AA, BagayokoMaiga K, Seraissol P, Darin K, Fraissinet F, Bahachimi A, Sanogo A, Lavit M, Chatelut E, Sylla M, Dao S, Murphy R, Gandia P. Evaluation of efavirenz and lopinavir concentrations in plasma and prediction of uptake by nursed infants in Mali. 17th International Workshop on Clinical Pharmacology of HIV \& Hepatitis; 2016 Jun 8-10; Washington DC.; Concentration d'Efavirenz et de lopinavir/r dans le lait maternel au Mali. Aboubacar Alassane Oumar, Bamako, Mali (Université des Sciences, des Techniques et des Technologies). 36th Interdisciplinary Meeting of Anti-Infectious Chemotherapy; 2016 Dec 12-13; Paris, France [Oral communication CO-021]; Oumar AA, BagayokoMaiga K, Darin K, Maiga M, Bahachimi A, Cere MC, Sanogo A, Chatelut E, Sylla M, Murphy R, Dao S, Gandia P. Evaluation du passage du Lopinavir/r dans le plasma et prédiction des quantités ingérées par le nourrisson allaité: étude chez la femme au Mali. 9th International Conference on AIDS and STIs in Africa; 2017 Dec 4-9; Abidjan, Ivory Coast [Oral communication S1164]; and Aboubacar Alassane Oumar, Kadiatou Bagayoko-Maiga, Mamoudou Maiga Aliou Bahachimi, Marie-Christine CERE, Zoumana Diarra, Alou Sanogo, Etienne Chatelut, Mariam Sylla, Robert Leo Murphy, Sounkalo Dao, Peggy GANDIA. Evaluation du Passage de l'Efavirenz dans le Lait Maternel et Prédiction des Quantités Ingérées par Le Nourrisson Allaité: Etude Chez la Femme au Mali. AFRAVIH 2018: 9th Edition of the International Francophone Conference on HIV/HEPATITIS; 2018 Apr 4-7; Bordeaux, France [Oral communication S14-01].

https://doi.org/10.1124/jpet.118.249938.

\section{Introduction}

The prevention of human immunodeficiency virus (HIV) transmission during pregnancy and breastfeeding remains a major public health problem for resource-limited countries (Joint United Nations Programme on HIV/AIDS, 2016; World Health Organization 2016). Since 2010, new HIV infections among children have decreased by half, but in 2015 still more than 150,000 children were newly infected with HIV worldwide (Joint United Nations Programme on HIV/AIDS, May, 31, 2016). The World Health Organization (2016) now recommends that all pregnant and breastfeeding women initiate lifelong antiretroviral therapy (ART), regardless of clinical stage or CD4 cell count, to improve women's health as well as to prevent the transmission of HIV from mother to child or to sexual partner. However, gaps in knowledge remain about the extent of transfer of maternal antiretroviral agents to infants throughout pregnancy and breastfeeding. Such information may be useful to identify optimal maternal antiretroviral regimens to maximize infant protection while minimizing toxicity.

The extent of antiretroviral passage from maternal plasma (MP) to breast milk (BM) and then to infant plasma (IP) varies 
considerably by antiretroviral drug class and individual agent. A recent systematic review of 24 studies (Waitt et al., 2015) that evaluated antiretroviral pharmacokinetics (PK) in breastfeeding, HIV-infected women reported a pooled point estimate for BM-to-MP ratio of 0.89 to 1.21 for the nucleoside reverse transcriptase inhibitors (14 studies, 1159 paired samples), 0.71 to 0.94 for the non-nucleoside reverse transcriptase inhibitors (17 studies, 965 paired samples), and 0.17 to 0.21 for the protease inhibitors (eight studies, 477 paired samples). In addition, some studies have evaluated efavirenz (EFV) concentrations in the BM of lactating HIV-infected mothers (Schneider et al., 2008; Olagunju et al., 2015; Palombi et al., 2016).

This particular work builds on and extends these prior studies with the goal of studying simultaneously and longitudinally (several time points in 6 months) the antiretroviral concentrations in BM and IP, the relationship between maternal antiretroviral PK and biologic parameters (viral load), as well as associated infant adverse effects. This study uniquely covers all these parameters together in the same population and at multiple time points.

\section{Materials and Methods}

Study Population. This was an observational, nonrandomized PK study among HIV-infected women (mothers) and their nursing infants from birth through 6 months postdelivery. Mother-infant pairs were recruited at the units of the Pediatric Department of the University Hospital Gabriel Touré (Bamako, Mali) and the common reference Health Center V (Bamako, Mali). The deliveries were uncomplicated. Women infected with HIV-1 were eligible for study enrollment during their last trimester of pregnancy or immediately postpartum if they were at least 18 years of age, residing in Bamako, receiving ART with EFV (600 mg once daily) or lopinavir (LPV)/ ritonavir (400/100 mg twice daily) and planned to breastfeed or were breastfeeding their infant. All women signed an informed consent form, and the study was approved by the ethics committee of Faculty of Medicine, Pharmacy and Dentistry of Mali.

PK Sampling. Maternal blood and BM samples and infant blood samples were collected at months 1,3 , and 6 postdelivery. Plasma and $\mathrm{BM}$ concentrations were determined at 12-14 hours postdose (middose interval) for EFV and approximately 1-2 hours after the mother's morning dose of LPV. For BM, mothers were instructed on a technique to hand express a sufficient quantity of BM into a collection device. The plasma was extracted and stored along with milk specimens in $-80^{\circ}$ freezers before being shipped cold to France for analysis. Adherence was assessed at each study visit; a study investigator asked each subject the exact time at which the last dose of EFV or LPV was taken, as well as whether any doses of ART were missed in the 1,2 , or 3 days prior to the study visit.

Laboratory and Clinical Assessment. Hematologic (blood count, hemoglobin), biochemical (alanine aminotransferase, creatinine, albumin), and immunologic (CD4 cell count) tests were evaluated at delivery in mothers and at the 1-, 3-, and 6-month postdelivery study visits in mothers and infants. The plasma HIV-1 RNA level was determined at delivery and 6 months postpartum for mothers and at 3 and 6 months postdelivery for infants using the Abbott M2000 RealTime HIV-1 assay with a lower limit of $40 \mathrm{copies} / \mathrm{ml}$. A physical examination of the mother (weight, height, temperature, palpation of the spleen) and infant (weight, size, temperature) were performed at each study visit. Also, at each postpartum study visit, mothers were actively asked to report any infant adverse effects (e.g., rash, neurodevelopmental delays)

Antiretroviral Pharmacokinetic Analysis. Plasma EFV and LPV concentrations were measured by liquid chromatographytandem mass spectrometry method. For calibration standards and quality control, $50 \mu \mathrm{g} / \mathrm{l}$ standard or quality control solutions were added into $200 \mu \mathrm{l}$ of internal standard (500 $\mathrm{ng} / \mathrm{ml}$ in acetonitrile) at the sample tube and vortexed immediately for 30 seconds. After 5 minutes of repose, sample tubes were revortexed and centrifuged for 10 minutes at $10,500 \mathrm{~g}$. Next, $50 \mu \mathrm{l}$ of supernatant was added to $200 \mu \mathrm{l}$ of liquid control quality water and vortexed for 5 seconds, and then $150 \mu \mathrm{l}$ of that solution was added to injection vials to liquid chromatography-tandem mass spectrometry (Applied Biosystems/MDS Sciex, Foster City, CA). The lower limit of quantification (LOQ) was set at $216 \mathrm{ng} / \mathrm{ml}$ for EFV and $264 \mathrm{ng} / \mathrm{ml}$ for LPV. Linearity ranged from LOQ to $4900 \mathrm{ng} / \mathrm{ml}$ for EFV and from LOQ to $11,400 \mathrm{ng} / \mathrm{ml}$ for LPV in all samples. The interassay precision as defined by CV ranged from $2.5 \%$ to $5.2 \%$ for EFV and from $4.5 \%$ to $3.9 \%$ for LPV.

A $25-\mu \mathrm{l}$ sample of BM was vortexed for 10 seconds before use and extracted with $1.5 \mathrm{ml}$ of methanol; this was vortexed for 60 seconds in the presence of internal standard. The extract was centrifuged at $3500 \mathrm{~g}$ for 10 minutes. The supernatant was transferred into a $5-\mathrm{ml}$ tube and evaporated to dryness in a rotary evaporator under vacuum (Thermo Electron Industries, Chateau Gontier, France) operating at $40^{\circ} \mathrm{C}$. The residue was reconstituted in $300 \mu \mathrm{l}$ of mobile phase $\mathrm{A}(2 \mathrm{mM}$ ammonium acetate, $0.2 \%$ acetic acid in water) and phase B $(2 \mathrm{mM}$ ammonium acetate, $0.2 \%$ acetic acid in methanol) $(40: 60, \mathrm{v} / \mathrm{v})$, and aliquots were transferred into autosampler vials. Linearity ranged from LOQ to $5000 \mathrm{ng} / \mathrm{ml}$ for EFV and LPV, and from LOQ to $1000 \mathrm{ng} / \mathrm{ml}$ for ritonavir in all samples. The interassay precision ranged from $3.5 \%$ to $7.8 \%$ for EFV and from $4.2 \%$ to $5.0 \%$ for LPV.

Relative Infant Dose. To estimate the ingested infant dose, we applied the following formula: ingested infant dose $(\mu \mathrm{g} / \mathrm{ml}$ per day $)=$ $\mathrm{C}_{\text {milk }} \times \mathrm{V}_{\mathrm{i}}$, where $\mathrm{C}_{\text {milk }}(\mu \mathrm{g} / \mathrm{ml})$ is the measured drug milk concentration and $V_{i}$ is the milk volume ingested by the infant, assuming an average volume of ingested $\mathrm{BM}$ of $150 \mathrm{ml} / \mathrm{kg}$ per day (Howard and Lawrence, 1999).

The relative infant dose received through BM can then be expressed as a percentage of the weight-adjusted maternal or pediatric dose, as follows: relative infant dose $\left(\%_{\text {maternal }}\right)=$ [ingested infant dose $(\mu \mathrm{g} / \mathrm{kg}$ per day)]/[maternal daily dose $(\mu \mathrm{g} / \mathrm{kg}$ per day $)] \times 100$, based on the median maternal weight at study baseline; or relative infant dose $\left(\%_{\text {pediatric }}\right)=[$ ingested infant dose $(\mu \mathrm{g} / \mathrm{kg})] /[$ pediatric daily dose $(\mu \mathrm{g} / \mathrm{kg}$ per day)] $\times 100$, based on the median infant weight at month 6 .

Infant Apparent Clearance. Apparent clearance is a hybrid PK parameter combining information from both drug absorption and elimination and bringing information on the PK behavior of a drug. From a PK point of view, apparent infant clearance (CL/F) is expressed as the following formula: $\mathrm{CL} / \mathrm{F}(\mathrm{l} / \mathrm{kg})=$ ingested dose $(\mu \mathrm{g} / \mathrm{kg}) / \mathrm{AUC}_{0-\mathrm{DI}}$, where AUC is the area under the concentrationtime curve for the dosing interval (DI). The ingested dose was calculated applying the previous formula, ingested infant dose $=$ $\mathrm{C}_{\text {milk }} \times \mathrm{V}_{\mathrm{i}}$, and the infant exposure $\left(\mathrm{AUC}_{0-\mathrm{DI}}\right)$ was calculated by multiplying the infant's plasma drug concentration by the DI following the PK formula: $\mathrm{AUC}_{0-\mathrm{DI}}=\mathrm{C}_{\text {plasma }} \times \mathrm{DI}$; $\left(A U C_{24 h}=C_{p-} 24 h\right)$, where $\mathrm{DI}=24$ hours for EFV and 12 hours for LPV. A breastfed infant's ingested dose and plasma AUC values were estimated from a single time point during the DI. Our computation assumes that a constant concentration of drug exists in BM and IP throughout the DI. This assumption seems reasonable for the following two reasons: 1) the drug concentration in both matrices (BM and breastfed IP) is supposed to be at steady state due to the repeated drug ingestion by the mother; and 2) a low variation of drug concentration is expected in ingested

ABBREVIATIONS: ART, antiretroviral therapy; AUC, area under the concentration-time curve; BM, breast milk; CL/F, apparent infant clearance; DI, dosing interval; EFV, efavirenz; HIV, human immunodeficiency virus; IP, infant plasma; IQR, interquartile range; LOQ, limit of quantification; LPV, lopinavir; MP, maternal plasma; TDF, tenofovir; ZDV, zidovudine. 
milk due to the short duration of breastfeeding $( \pm 20$ minutes of suckling) as well as in breastfed IP due to repeated sucklings throughout the DI (6-8 sucklings/day), mimicking a perfusion.

Statistical Analysis. Mother-infant demographics and laboratory parameters were summarized descriptively by frequency or proportions and as mean (S.D.) or median [interquartile range (IQR)], as appropriate. EFV and LPV concentrations in MP, BM, and IP were reported as median with IQR and the ratio of median $\mathrm{BM} / \mathrm{MP}$ values for each study time point. The relative infant dose and the apparent clearance were reported as the mean (S.D.) and median (IQR), respectively. Reported infant adverse effects were graded according to the Division of AIDS (DAIDS) Table for Grading the Severity of Adult and Pediatric Adverse Events (version 2.0; November 2014) (https://rsc.tech-res.com/docs/default-source/safety/ daids_ae_grading_table_v2_nov2014.pdf?sfvrsn=8) and reported using appropriate descriptive statistics. Adverse effects were categorized as mild to moderate (grades 1 or 2 ) or severe to potentially life threatening (grades 3 or 4). A Fisher's exact test or chi square test was used, as appropriate, to evaluate the association between EFV or LPV concentration and HIV-RNA level or the presence of infant adverse effects.

\section{Results}

From May 2014 to January 2016, 48 mother-infant pairs were enrolled into the study and 41 pairs completed the study. Seven women withdrew from the study because they stopped breastfeeding before month 3; their data are not reported. Mother and infant characteristics are summarized in Table 1. The mean (S.D.) mothers' age was 29 years (5.3 years). During pregnancy, mothers were prescribed a three-drug ART regimen that was continued during breastfeeding; 32 mothers received EFV plus tenofovir (TDF) and lamivudine; while 9 received lopinavir/ritonavir plus zidovudine (ZDV) and lamivudine. The median (IQR) duration of ART before study inclusion was 57 months ( $0-168$ months); all women reported $100 \%$ adherence to ART based on diary cards for 3 days prior to the study visit. Each mother enrolled in the study gave birth to one infant.

EFV Pharmacokinetics and Infant Exposure. Among the 32 maternal-infant pairs exposed to EFV who completed the study, there were $83 \mathrm{MP}(86 \%), 82 \mathrm{IP}(85 \%)$, and $83 \mathrm{BM}$ (86\%) samples available for analysis across all three study time points; samples were excluded from the final analysis due to a shipping error. Median MP and BM concentrations, as well as IP concentrations, of EFV are reported in Table 2. Across the study period, the median (IQR) BM/MP ratio of EFV was 1.16 (0.96-20.62), which corresponds to a relative infant dose of $2.46 \%$ of the recommended weight-adjusted pediatric EFV dose at month 6 . The median (IQR) $\mathrm{CL} / \mathrm{F}$ values of EFV were 0.098 (0.076-0.198), 0.085 (0.075-0.139), and $0.146(0.111-0.246) \mathrm{l} / \mathrm{h}$ per kilogram at months 1,3 , and 6 , respectively.

LPV Pharmacokinetics and Infant Exposure. Among the nine maternal-infant pairs exposed to LPV/ritonavir who participated in the study, there were $21 \mathrm{MP}$ (78\%), 20 IP (74\%), and $23 \mathrm{BM}(85 \%)$ samples available for analysis across all three study time points. Median MP and BM concentrations, as well as IP concentrations, of LPV are reported in Table 3. Across the study period, the median (IQR) BM/MP ratio of LPV was $0.063(0.036-0.088)$, which corresponds to an estimated relative infant dose of $0.23 \%$ of the recommended weight-adjusted pediatric LPV dose at month 6 .
TABLE 1

Participants' characteristics

Data presented as the mean (S.D.), unless otherwise indicated.

\begin{tabular}{lcc}
\hline \multicolumn{1}{c}{ Characteristic } & Mothers $(n=41)$ & Infants $(n=41)$ \\
\hline Age $(\mathrm{yr})$ & $29(5.3)$ & \\
Weight $(\mathrm{kg})$ & $56(7.2)$ & \\
HIV-1 RNA $<40$ copies $/ \mathrm{ml}, n(\%)$ & $36(88)$ & \\
Mean CD4 ${ }^{+}$count $\left(\right.$cells $\left./ \mathrm{mm}^{3}\right)$ & $588(260)$ & $37.1(0.3)$ \\
Gestational age at birth $(\mathrm{wk})$ & & $3992(506)$ \\
Weight at birth $(\mathrm{g})$ & & $6183(624)$ \\
Weight at month $6(\mathrm{~g})$ & & \\
\hline
\end{tabular}

Safety Considerations. At delivery, five women (12\%) had detectable HIV RNA levels in plasma (mean, 2467 copies/ml; S.D., 3122 copies $/ \mathrm{ml}$ ). At 6 months postdelivery, two of these same women had persistent viremia (HIV RNA levels of 593 and 200 copies/ml). There was no significant difference between drug concentrations in women with or without detectable HIV RNA. All infants were HIV negative at birth and remained HIV negative throughout the study period.

During the 6 months of follow-up after birth, there were four occurrences of grades 1 or 2 anemia among the 41 infants; 32 infants were exposed to EFV, TDF, and lamivudine; and 9 infants were exposed to LPV/ritonavir, ZDV, and lamivudine. No severe anemia (grades 3 or 4) in breastfed infants were found in this study. Overall, there was no difference in the median hemoglobin levels at month 1 versus month 6 (11.1 vs. $12.1 \mathrm{~g} / \mathrm{dl})$ in infants born to women in treatment $(P=0.56)$. There were no other drug-related adverse effects identified or reported in the infants throughout the study period.

\section{Discussion}

This study explored the extent to which EFV and LPV are excreted from MP to BM and to IP in Malian mothers living with HIV and their paired nursing infants, and also explored associations between antiretroviral exposure and safety parameters. Among 32 mother-infant pairs, EFV was efficiently excreted into the $\mathrm{BM}$, represented by a $\mathrm{BM} / \mathrm{MP}$ ratio ranging from 1.06 to 1.27 during the follow-up at 6 months postdelivery. However, the subsequent transfer of EFV from BM to IP was low; IP/MP ratios ranged from 0.048 to 0.072 over the same time period. Further to this, we calculated that nursing infants ingested less than $3 \%$ of the relative infant dose of EFV from BM, based on a weight-adjusted recommended pediatric dose (https://aidsinfo.nih.gov/contentfiles/lvguidelines/pediatricguidelines.pdf). In contrast to EFV, LPV was inefficiently excreted into the $\mathrm{BM}$, represented by $\mathrm{BM} / \mathrm{MP}$ ratios ranging from 0.088 to 0.39 over 6 months of follow-up. Based on this exposure in BM, we calculated a relative infant dose of $0.23 \%$ of the recommended pediatric dose of LPV, adjusted for weight in a 6-month-old infant. This estimated negligible transfer of LPV from mother-to-infant via BM was supported by measured IP concentrations, which were undetectable in all infants over the 6 months postdelivery.

In the study of the passage of EFV in BM, our results are consistent with previous studies Schneider et al., 2008; Olagunju et al., 2015; Palombi et al., 2016; ter Heine et al., 2008. $(5-7,17)$ showing high concentrations of this drug in BM and low levels in breastfeeding infants. For EFV, using an intensive PK study design over the 24-hour DI, Olagunju et al. (2015) reported a median (range) $\mathrm{BM} /$ plasma ratio of 1.10 
TABLE 2

EFV concentration (nanograms per milliliter) in MP, BM, and IP over 6 months postpartum

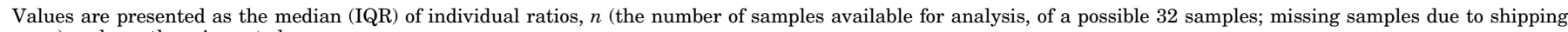
error), unless otherwise noted.

\begin{tabular}{|c|c|c|c|c|c|c|}
\hline Study Visit & $\mathrm{MP}^{a}$ & $\mathrm{BM}^{b}$ & $\mathrm{IP}^{c}$ & BM/MP Ratio & IP/MP Ratio & Relative Infant Dose \\
\hline & & & & & & $\%$ \\
\hline Month 1 & $\begin{array}{c}2930(2450-6595) \\
n=22\end{array}$ & $\begin{array}{c}4660(2940-7660) \\
n=22\end{array}$ & $\begin{array}{c}347(282-573), \\
n=22\end{array}$ & $\begin{array}{c}1.11(0.89-1.73), \\
n=19\end{array}$ & $\begin{array}{c}0.057(0.03-0.11) \\
n=20\end{array}$ & \\
\hline Month 3 & $\begin{array}{c}3050(2060-6685) \\
n=31\end{array}$ & $\begin{array}{c}4160(1900-6440) \\
n=31\end{array}$ & $\begin{array}{c}268(142-465), \\
n=30\end{array}$ & $\begin{array}{c}1.06(0.86-1.63), \\
n=30\end{array}$ & $\begin{array}{c}0.072(0.05-0.09) \\
n=28\end{array}$ & \\
\hline Month 6 & $\begin{array}{c}2740(2150-8080) \\
n=30\end{array}$ & $\begin{array}{c}3920(2570-9350) \\
n=30\end{array}$ & $\begin{array}{c}175(90-331) \\
n=30\end{array}$ & $\begin{array}{c}1.27(0.96-1.50), \\
n=28\end{array}$ & $\begin{array}{c}0.048(0.03-0.07) \\
n=24\end{array}$ & 2.46 \\
\hline
\end{tabular}

${ }^{a}$ MP EFV was below the LOQ in one sample each at months 1,3 , and 6.

${ }^{b} \mathrm{BM}$ EFV was below LOQ in one sample at months 1 and 3 , and in two samples at months 6 .

${ }^{c}$ IP EFV was below LOQ in one, two, and three samples at months 1,3 , and 6 , respectively.

(0.57-1.71) among 29 mother-infant pairs. Also similar to our study, Olagunju et al. (2015) calculated the average exposure index, relative to a weight-adjusted pediatric and maternal EFV dose, which they reported as a median (range) of $2.14 \%$ $(0.82 \%-8.05 \%)$ and $7.69 \%(2.04 \%-26.2 \%)$, respectively. This compares to our findings of $2.46 \%$ and $6.6 \%$, respectively, for relative infant dose to weight-adjusted pediatric and maternal doses of EFV. In another study, Palombi et al. (2016), described a median (IQR) BM/MP ratio of $0.76(0.50-1.10)$ at 1-month postpartrum and 0.86 (0.54-1.13) among 33 mothers (month 1), 47 mothers (month 12), and 25 infants in Malawi. Other authors found results lower than ours (Schneider et al., 2008; in Rwanda 0.54).

The passage of the drug into $\mathrm{BM}$ is determined by the $\mathrm{PK}$ characteristics of the mother, the amount excreted in BM, and the amount ingested by the infant (Anderson, 2006). High interindividual variability and intraindividual variability in EFV PK has been observed in children (Brundage et al., 2004).

The first study was conducted in Rwanda ( $n=13$ motherinfant pairs) that reported a $\mathrm{BM} / \mathrm{MP}$ ratio of 0.54 and an IP/BM ratio of 0.24 , which corresponds to an infant EFV concentration that is approximately $13 \%$ of maternal concentrations; no infant adverse effects were reported (Schneider et al., 2008). The second study, conducted in Nigeria ( $n=$ 29 mother-infant pairs), reported a median (range) BM/MP ratio of $1.10(0.57-1.71)$ and a median average (range) infant dose of 428 (164-1610) $\mu \mathrm{g} / \mathrm{kg}$ per day, which represents $4.05 \%$ (1.08-13.8) of a weight-adjusted pediatric dose (Olagunju et al., 2015). The third study was conducted in Malawi ( $n=$ 47 mothers and 25 infants) and reported a median (IQR) $\mathrm{BM} / \mathrm{MP}$ ratio of $0.76(0.50-1.10)$ and $0.86(0.54-1.13)$, respectively, at month 1 and month 12 of infant age (Palombi et al., 2016).
Two previous studies (Palombi et al., 2012; Corbett et al., 2014) evaluated LPV concentrations in BM and infant feeding with very low concentrations. Our results confirm the previous results on the low passage of LPV in human milk. We reported a BM/MP ratio of 0.063 of LPV. This result is lower than that of Palombi et al. (2012), with 0.38 and Corbett et al. (2014), with 0.54. However, these differences can be explained by the difference in the methodology used. Indeed, Palombi et al. (2012) used a method with an LOQ at $10 \mathrm{ng} / \mathrm{ml}$, which is much lower than that determined using our method $(216 \mathrm{ng} / \mathrm{ml})$. We estimated a relative child dose at $0.23 \%$. Few studies have been interested in the relative infant dose of LPV. The high protein binding for LPV could explain this low diffusion in milk (Shapiro et al., 2013).

EFV is mainly metabolized in the liver by the enzymes CYP2B6, CYP2A6, CYP3A4, and UGT2B7 (Olagunju et al., 2012). Salem et al. (2014) found that $90 \%$ of the maturity of EFV metabolism was not reached until 9 months of age. This may partly explain the lower apparent clearances that we estimated for breastfed newborns compared with those reported in children $(0.19 \mathrm{l} / \mathrm{h}$ per kilogram; 0.21-0.26) (Fletcher et al., 2008) and $0.30 \mathrm{l} / \mathrm{h}$ per kilogram (0.07-1.0) (Hoody and Fletcher, 2003) and in adults $(0.15 \mathrm{l} / \mathrm{h}$ per kilogram).These results seem to be explained by the fact that EFV is mainly metabolized by the enzyme CYP450 2B6, the expression of which increases with age (Pearce et al., 2016). However, CYP2B6 expression does not completely explain the fact that adults present lower apparent clearances compared with children.

We found a plasma concentration of EFV in the infant of $356 \mathrm{ng} / \mathrm{ml}$ at month $1,268 \mathrm{ng} / \mathrm{ml}$ at month 3 , and $175 \mathrm{ng} / \mathrm{ml}$ at month 6 . This result is similar to previous reports from other studies. Using a 3-day medication diary, which showed

TABLE 3

LPV concentration (nanograms per milliliter) in MP, BM, and IP over 6 months postpartum

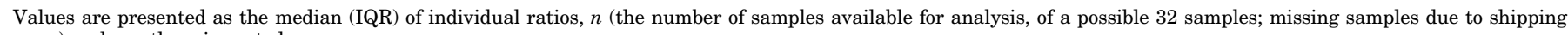
error), unless otherwise noted.

\begin{tabular}{|c|c|c|c|c|c|c|}
\hline Study Visit & $\mathrm{MP}^{a}$ & $\mathrm{BM}^{b}$ & $\mathrm{IP}^{c}$ & BM/MP Ratio & IP/MP Ratio ${ }^{d}$ & $\begin{array}{c}\text { Relative } \\
\text { Infant Dose }\end{array}$ \\
\hline & & & & & & $\%$ \\
\hline Month 1 & $1870(586-4190) n=4$ & $530(150-890) n=6$ & $<\operatorname{LOQ} n=3$ & $0.39(0.07-0.62)$ & & \\
\hline Month 3 & $10,900(5495-15,750) n=9$ & $650(160-940) n=8$ & $<\operatorname{LOQ} n=9$ & $0.056(0.036-0.064)$ & & \\
\hline Month 6 & $5790(1230-10,600) n=8$ & $590(200-770) n=8$ & $<\operatorname{LOQ} n=8$ & $0.088(0.06-0.14)$ & & 0.23 \\
\hline
\end{tabular}

${ }^{a}$ MP LPV was below the LOQ in one sample in months 1 and 6.

${ }^{b}$ BM LPV was below the LOQ in one sample in months 1 and 3 , and in two samples in month 6 .

${ }^{c}$ IP LPV was below the LOQ in three, eight, and seven samples in months 1, 3, and 6, respectively.

${ }^{d}$ LPV concentration fell below the LOQ for all IP samples; therefore, the IP/MP ratio was not calculated. 
$100 \%$ self-reported observance of our study subjects, we found no association between adherence and PK parameters. However, despite a good adherence of the mothers, we observed two mothers with undetectable plasma levels at month 6 .

None of the infants had a clinical adverse effect related to their mother's treatment. However, four children had minor hematologic adverse reactions of grade 1 and 2 up to month 6 of the follow-up, and there were differences according to the treatment of their mother (TDF vs. ZDV). No adverse reactions or toxicity related to antiretroviral agents taken by the mother was observed in children. Gandhi et al. (2013) have described some adverse effects of hematologic type of grade 1 or 2 in their study. Severe anemia occurred in 11 of 38 infants (28.9\%) exposed to ZDV (Palombi et al., 2012). In our study, the majority of mothers (32 of 41) received TDF; only 9 mothers were taking ZDV. The microcytic anemia is frequent in infants. The mean value varies from the first month to the sixth month of life (Palombi et al., 2012). We did not find cases of severe anemia in the study. Although Dryden-Peterson et al. (2011) reported a higher incidence of severe anemia in infants exposed to ZDV-based maternal ART in utero; our study did not find an association. In addition, there was no difference between infants exposed to ZDV and those exposed to TDF. Bae et al. (2008) reported increased the risk of neutropenia up to 1 month of age after in utero exposure to maternal ART. In our study, there were no cases of severe neutropenia. However, we cannot rule out a previous effect of exposure to ART since our first test was performed at 1 month of age. Our study has several limitations. The usefulness of monitoring the effect of antiretroviral agents present in milk on the virus present in milk is important to prevent resistance in breastfed infants. Giuliano et al. (2007) reported that triple therapy [azidothymidine, 3TC (lamivudine), and nevirapine] administered during pregnancy and postpartum significantly reduced the viral load in BM, thereby reducing viral exposure across newborns breastfed with BM. In this study, all children have EFV concentrations in plasma below the recommended therapeutic level for adults. In the case of HIV infection, exposure to subtherapeutic concentrations of EFV through BM may promote resistance of the virus, as described by other authors (Zeh et al., 2011; Inzaule et al., 2016).

In conclusion, in our study, we have shown that during ART in breastfeeding women, EFV is detectable at low levels in the plasma of breastfed infants and without associated side effects. However, LPV was undetectable in the plasma of breastfed infants. In addition, the mothers had good virologic responses during ART, and all breastfed infants remained HIV negative throughout the 6-month study period. Further studies are needed to evaluate the longterm safety concerns of infants exposed to EFV beyond 6 months of breastfeeding.

\section{Acknowledgments}

We thank the patients, staff, and management at the units of the Pediatric Department of the University Hospital Gabriel Touré and the common reference Health Center V, in Mali who participated in this study for their support. We also thank the staff at the Toulouse Pharmacokinetic Laboratory Facility for their input during the development and validation of the method Dr. Francois
Fraissinet, Dr. Michel Lavit, M. Patrick Seraissol, M. Alou Sanogo, Pr. Bruno Marchou, Dr. Kritin Michelle Darin, Pr. Souleymane Diallo.

\section{Authorship Contributions}

Participated in research design: Oumar and Gandia.

Conducted experiments: Oumar, Bagayoko-Maiga, Bahachimi, Maiga, Cere, Diarra, Sylla, Murphy, and Dao.

Performed data analysis: Oumar, Chatelut, Dao, Gandia.

Wrote or contributed to the writing of the manuscript: Oumar, Bagayoko-Maiga, Bahachimi, Maiga, Cere, Diarra, Chatelut, Sylla, Murphy, Dao, and Gandia.

\section{References}

Anderson GD (2006) Using pharmacokinetics to predict the effects of pregnancy and maternal-infant transfer of drugs during lactation. Expert Opin Drug Metab Toxicol 2:947-960.

Bae WH, Wester C, Smeaton LM, Shapiro RL, Lockman S, Onyait K, Thior I, and Essex M (2008) Hematologic and hepatic toxicities associated with antenatal and postnatal exposure to maternal highly active antiretroviral therapy among infants. AIDS 22:1633-1640.

Brundage RC, Yong FH, Fenton T, Spector SA, Starr SE, and Fletcher CV (2004) Intrapatient variability of efavirenz concentrations as a predictor of virologic response to antiretroviral therapy. Antimicrob Agents Chemother 48:979-984.

Corbett AH, Kayira D, White NR, Davis NL, Kourtis AP, Chasela C, Martinson F, Phiri G, Musisi B, Kamwendo D, et al.; BAN Study Team (2014) Antiretroviral pharmacokinetics in mothers and breastfeeding infants from 6 to 24 weeks postpartum: results of the BAN Study. Antivir Ther 19:587-595.

Dryden-Peterson S, Shapiro RL, Hughes MD, Powis K, Ogwu A, Moffat C, Moyo S, Makhema J, Essex M, and Lockman S (2011) Increased risk of severe infant anemia after exposure to maternal HAART, Botswana. J Acquir Immune Defic Syndr 56:428-436.

Fletcher CV, Brundage RC, Fenton T, Alvero CG, Powell C, Mofenson LM, and Spector SA (2008) Pharmacokinetics and pharmacodynamics of efavirenz and nelfinavir in HIV-infected children participating in an area-under-the-curve controlled trial. Clin Pharmacol Ther 83:300-306.

Gandhi M, Mwesigwa J, Aweeka F, Plenty A, Charlebois E, Ruel TD, Huang Y, Clark T, Ades V, Natureeba P, et al.; Prevention of Malaria and HIV disease in Tororo (PROMOTE) study (2013) Hair and plasma data show that lopinavir, ritonavir, and efavirenz all transfer from mother to infant in utero, but only efavirenz transfers via breastfeeding. J Acquir Immune Defic Syndr 63:578-584.

Giuliano M, Guidotti G, Andreotti M, Pirillo MF, Villani P, Liotta G, Marazzi MC, Mancini MG, Cusato M, Germano P, et al. (2007) Triple antiretroviral prophylaxis administered during pregnancy and after delivery significantly reduces breast milk viral load: a study within the drug resource enhancement against AIDS and malnutrition program. J Acquir Immune Defic Syndr 44:286-291.

Hoody DW and Fletcher CV (2003) Pharmacology considerations for antiretroviral therapy in human immunodeficiency virus (HIV)-infected children. Semin Pediatr Infect Dis 14:286-294.

Howard CR and Lawrence RA (1999) Drugs and breastfeeding. Clin Perinatol 26 447-478.

Inzaule SC, Weidle PJ, Yang C, Ndiege K, Hamers RL, Rinke de Wit TF, Thomas T, and Zeh C (2016) Prevalence and dynamics of the K65R drug resistance mutation in HIV-1-infected infants exposed to maternal therapy with lamivudine, zidovudine and either nevirapine or nelfinavir in breast milk. J Antimicrob Chemother 71:1619-1626.

Olagunju A, Bolaji O, Amara A, Waitt C, Else L, Adejuyigbe E, Siccardi M, Back D, Khoo S, and Owen A (2015) Breast milk pharmacokinetics of efavirenz and breastfed infants' exposure in genetically defined subgroups of mother-infant pairs: an observational study. Clin Infect Dis 61:453-463.

Olagunju A, Owen A, and Cressey TR (2012) Potential effect of pharmacogenetics on maternal, fetal and infant antiretroviral drug exposure during pregnancy and breastfeeding. Pharmacogenomics 13:1501-1522.

Palombi L, Pirillo MF, Andreotti M, Liotta G, Erba F, Sagno JB, Maulidi M, Ceffa S, Jere H, Marchei E, et al. (2012) Antiretroviral prophylaxis for breastfeeding transmission in Malawi: drug concentrations, virological efficacy and safety. Antivir Ther 17:1511-1519.

Palombi L, Pirillo MF, Marchei E, Jere H, Sagno JB, Luhanga R, Floridia M, Andreotti M, Galluzzo CM, Pichini S, et al. (2016) Concentrations of tenofovir, lamivudine and efavirenz in mothers and children enrolled under the Option B-Plus approach in Malawi. J Antimicrob Chemother 71:1027-1030.

Pearce RE, Gaedigk R, Twist GP, Dai H, Riffel AK, Leeder JS, and Gaedigk A (2016) Developmental expression of CYP2B6: a comprehensive analysis of mRNA expression, protein content and bupropion hydroxylase activity and the impact of genetic variation. Drug Metab Dispos 44:948-958.

Salem AH, Fletcher CV, and Brundage RC (2014) Pharmacometric characterization of efavirenz developmental pharmacokinetics and pharmacogenetics in HIVinfected children. Antimicrob Agents Chemother 58:136-143.

Schneider S, Peltier A, Gras A, Arendt V, Karasi-Omes C, Mujawamariwa A Ndimubanzi PC, Ndayisaba G, and Wennig R (2008) Efavirenz in human breast milk, mothers', and newborns' plasma. J Acquir Immune Defic Syndr 48: 450-454.

Shapiro RL, Rossi S, Ogwu A, Moss M, Leidner J, Moffat C, Lockman S, Moyo S, Makhema J, Essex M, et al. (2013) Therapeutic levels of lopinavir in late pregnancy 
and abacavir passage into breast milk in the Mma Bana Study, Botswana. Antivir Ther 18:585-590.

Joint United Nations Programme on HIV/AIDS (2016) Global AIDS Update 2016, in UNAIDS / WHO (2016) Global Report. UNAIDS Report on the Global AIDS Epidemic 2016, UNAIDS, Geneva, Switzerland.

Waitt CJ, Garner P, Bonnett LJ, Khoo SH, and Else LJ (2015) Is infant exposure to antiretroviral drugs during breastfeeding quantitatively important? A systematic review and meta-analysis of pharmacokinetic studies. J Antimicrob Chemother $\mathbf{7 0}$ : 1928-1941.

World Health Organization (2016) Consolidated Guidelines on the Use of Antiretroviral Drugs for Treating and Preventing HIV Infection, 2nd ed, World Health Organization, Geneva, Switzerland.
Zeh C, Weidle PJ, Nafisa L, Lwamba HM, Okonji J, Anyango E, Bondo P, Masaba R, Fowler MG, Nkengasong JN, et al. (2011) HIV-1 drug resistance emergence among breastfeeding infants born to HIV-infected mothers during a single-arm trial of triple-antiretroviral prophylaxis for prevention of mother-to-child transmission: a secondary analysis. PLoS Med 8:e1000430.

Address correspondence to: Aboubacar Alassane Oumar, HIV/TB Research and Training Center (SEREFO), University of Sciences, Techniques and Technologies of Bamako (USTTB), Bamako BP. 1805, Mali. E-mail: aao@ icermali.org 